\title{
The 100 top-cited articles on scrub typhus: a bibliometric analysis
}

\author{
Taha Hussein Musa ${ }^{1,2 \oplus}$, Wei $\mathrm{Li}^{1}{ }^{1 \oplus}$, Joseph Kawuki $^{3 \oplus}$, Pingmin Wei ${ }^{1 \oplus}$ \\ ${ }^{1}$ Key Laboratory of Environmental Medicine Engineering, Ministry of Education, Department of Epidemiology and Health Statistics, \\ School of Public Health, Southeast University, Nanjing, China \\ ${ }^{2}$ Biomedical Research Institute, Darfur College, Nyala, Sudan \\ ${ }^{3}$ Centre for Health Behaviours Research, Jockey Club School of Public Health and Primary Care, Faculty of Medicine, The Chinese \\ University of Hong Kong, Hong Kong, China
}

Received: October 15, 2020

Revised: December 29, 2020

Accepted: January 27, 2021

Corresponding author:

Pingmin Wei

Key Laboratory of

Environmental Medicine

Engineering, Ministry of

Education, Department of

Epidemiology and Health

Statistics, School of Public

Health, Southeast University,

Nanjing, Jiangsu 210009,

China

E-mail: mpw1963@126.com

Co-Corresponding author:

Taha Hussein Musa

Key Laboratory of

Environmental Medicine

Engineering, Ministry of

Education, Department of

Epidemiology and Health

Statistics, School of Public

Health, Southeast University,

Nanjing, Jiangsu 210009,

China

E-mail: taha.hm99@yahoo.com

\section{ABSTRACT}

Objectives: The aims of this study were to analyze the characteristics of the 100 top-cited articles on scrub typhus (ST), and to assess the present research landscape and future research directions using bibliometric analysis.

Methods: Web of Science was used to conduct a bibliometric analysis of the 100 top-cited articles on ST. The articles were analyzed by publication year, number of citations, document type, journals, keywords, institutions, country of origin, and authorship.

Results: The top 100 articles on ST were published between 1945 to 2017. The number of citations ranges from 39 to 227 and the interquartile range was 35.5. The United States published the highest number $(n=21)$ of articles. Mahidol University was the most prolific institution in terms of articles $(n=14)$. The American Journal of Tropical Medicine and Hygiene was the journal with the most articles $(n=14)$, and Paris DH was the most productive author in terms of the Hirsh-index, which was 10 for that author. The study revealed a significant correlation between the total number of citations and the number of authors $(r=0.668$, $p<0.001)$, number of institutions $(r=0.692, p<0.001)$, number of years since publication $(r=0.869, p<0.001)$, and number of countries involved $(r=0.963, p<0.001)$.

Conclusion: The findings of this study provide landmarks in the publication and citation frequency of the most influential articles on ST. In addition, this study provides useful information for readers and health policy-makers in evaluating the literature on ST.

Keywords: Bibliometrics; Citation analysis; Scrub typhus

\section{Introduction}

Scrub typhus (ST), also known as tsutsugamushi disease [1], is a frequently neglected tropical disease most often transmitted through the bites of chiggers in rural areas [2]. The disease has been reported to be responsible for causing acute febrile illness, which is a serious public 
health burden throughout the wide endemic region known as the "tsutsugamushi triangle" [3].

More than 1 billion residents of rural areas across all age groups are estimated to be at risk, with approximately 1 million new cases reported annually [4,5]. Although there has been marked progress in antibiotics such as doxycycline, tetracycline, and chloramphenicol, which serve as the firstline treatment for ST, the risk of ST infection and reported cases have increased every year [6]. This suggests an urgent need for novel research regarding the development of an effective antibiotic and vaccine for the prevention and control of ST [7].

Bibliometric analysis can provide a macroscopic overview of a large volume of scientific literature in terms of historical background and help identify the most influential authors, journals, countries, institutions, and references in the historical development of scientific research in any field [8]. The use of bibliometrics as a technique can also help to identify a variety of research themes, illuminate the multidisciplinary character of a research domain, and determine research gaps, future research directions, and leading trends within in any particular field [9]. In addition, the technique enables assessment of active and influential authors, journals, countries, organizations and institutions in a given field, as well as annual publication trends [10].

Although there has been an increase in the amount of scientific research on ST, the disease is still remarkably neglected relative to other tropical diseases [11]. Today, more than 80 years after the end of the Second World War, there is still no effective human vaccine against ST infection [12], which may be due to the multiple antigenic strains of Orientia tsutsugamushi [13]. Thus, we applied bibliometric methods, which have been widely used by researchers across diverse scientific research fields, to assess and evaluate the development of the field and research trends in the study of ST since the end of World War II [9,14,15].

The purpose of this study was to perform a bibliometric analysis of the 100 most-cited articles on ST indexed in the Web of Science (WoS) from 1900 to December 2020. To our knowledge, this is the first bibliometric study conducted on the 100 most-cited ST articles.

\section{Materials and Methods}

\section{Study Design and Data Source}

We used the results of a search within the WoS database (Clarivate Analytics, Philadelphia, PA, USA) for the baseline data used in this study $[16,17]$. Bibliometric analyses have been undertaken in various research fields to monitor and evaluate the growth of scientific output and to provide support for designing suitable policies and making public health decisions [15-17]. Science Citation Index Expanded (SCIE) and Science Citation Index (SCI) were both used to identify the top 100 most-cited articles on ST.

\section{Search methodology}

As in other bibliometric studies [10,18], the overall searchz was conducted in a single day to avoid the possibility of daily citation updates to the database interfering with the results and leading to minor statistical changes over time [19]. Our search strategy was to use specific keywords on ST, which were obtained from the Medical Subject Headings (MeSH) index of biomedical literature (https:// meshb.nlm.nih.gov/search). We restricted our search to the presence of keywords in the title (titles including: "Orientia tsutsugamushi infection*" or "Tsutsugamushi disease*" or "Tsutsugamushi fever" or "Scrub typhus") and used the largest time span allowed in the WoS search function ("all years [1990-2020]") (search date December 10, 2020). Only original articles and reviews were used in this study, while editorials, proceedings, corrections, meeting abstracts, news items, letters, notes, early access publications, and reprints were excluded from our final dataset.

The search was restricted to articles published in English. The entire retrieval and data extraction process was conducted by 2 independent researchers (THM, JK).

The eligible articles were arranged in decreasing order of their citation count, after which the 100 top-cited articles were downloaded in CSV format, plain text format, and tabdelimited (win) format for further analysis. The following characteristics were considered in our analysis: publication year, citation count, authorship, affiliation (institution and country), journal name, and keywords. The journal impact factor (JIF) for each journal was obtained from WoS through manual search.

\section{Statistical Analysis}

Bibliometric analysis was used to examine the production of scientific research in the ST field. The distribution of the most productive countries, institutions, authors, journals, and keywords, as well as impact factor and total global citations, were analyzed using HistCite and bibliometrix, an $\mathrm{R}$ tool for comprehensive science mapping analysis [20]. Research collaborations between authors, countries, and organizations were mapped using VoSviewer ver. 1.6.6 [21]. Given the distribution of variables and the presence of outliers, we preferred to use the median, range, and interquartile range (IQR) to describe some variables since these measures are less affected by extreme values [10].

For citation analysis, the Hirsh-index (h-index) was used, 
which is a measure of both the number of publications on ST and the number of citations they received, with higher $\mathrm{h}$-index values suggesting a higher impact [19]. In addition, GraphPad Prism ver. 6 (GraphPad Software, San Diego, CA, USA) and IBM SPSS ver. 25.0 (IBM Corp., Armonk, NY, USA) were used for the statistical analysis. Spearman correlation coefficients were used to evaluate the associations between the number of citations and year of publication, authors, institutions, JIF, and countries. $p$ of less than 0.05 were considered to indicate statistical significance.

\section{Results}

\section{General Information}

The study identified 1,217 articles from 1900 to 2020 during the initial WoS search, and the 100 top-cited articles were identified after restricting the results to articles written in English. The majority of articles in the top 100 were original research articles $(n=88)$, with a total of 5,632 citations. The remaining entries were reviews $(n=12)$, which had a total of 392 citations. Altogether they had an h-index of 63. Publication years ranged from 1945 to 2017. The 100 mostcited articles were published in 49 journals by authors from 21 countries. In total, 392 authors, approximately 3.92 per document, contributed to the top 100 articles. The general characteristics of the metadata are presented in Table S1.

\section{Years of Publication}

As seen in Figure 1, a continuous upward trend was observed in the citation of ST articles after 1975. The number of articles increased along with the number of citations reported for each year during the study period. Among the top 100 articles, the majority $(n=63)$ were published between 1990 and 2017, with the greatest number of highly cited ST articles $(n=40)$ being published between 2005 and 2017.

\section{The 100 Most-Cited Articles}

The 100 most-cited articles in the field of ST and the number of citations, year of publication, and adjusted citation index (ACI) are presented in Table S2. The overall number of citations of the 100 most-cited articles was 6,994 , with a median of 56, range of 39 to 227, and IQR of 35.5. According to the global citations, the top-ranking article was published in 2003 by Watt and Parola [1] on ("Scrub Typhus and Tropical Rickettsioses"), followed by the 2009 article of Kelly et al. [3] entitled "Scrub Typhus: The Geographic Distribution of Phenotypic and Genotypic Variants of tsutsugamushi," both of which were cited more than 200 times.

In addition, the 100 most cited articles were indexed in many research categories including Public, Environmental and Occupational Health; Tropical Medicine; General and Internal Medicine; Microbiology; and Pathology among others.

\section{Journal Citation and Impact Factor Analysis}

The top 100 articles were published in 49 different journals. Fourteen journals that published at least 2 articles are

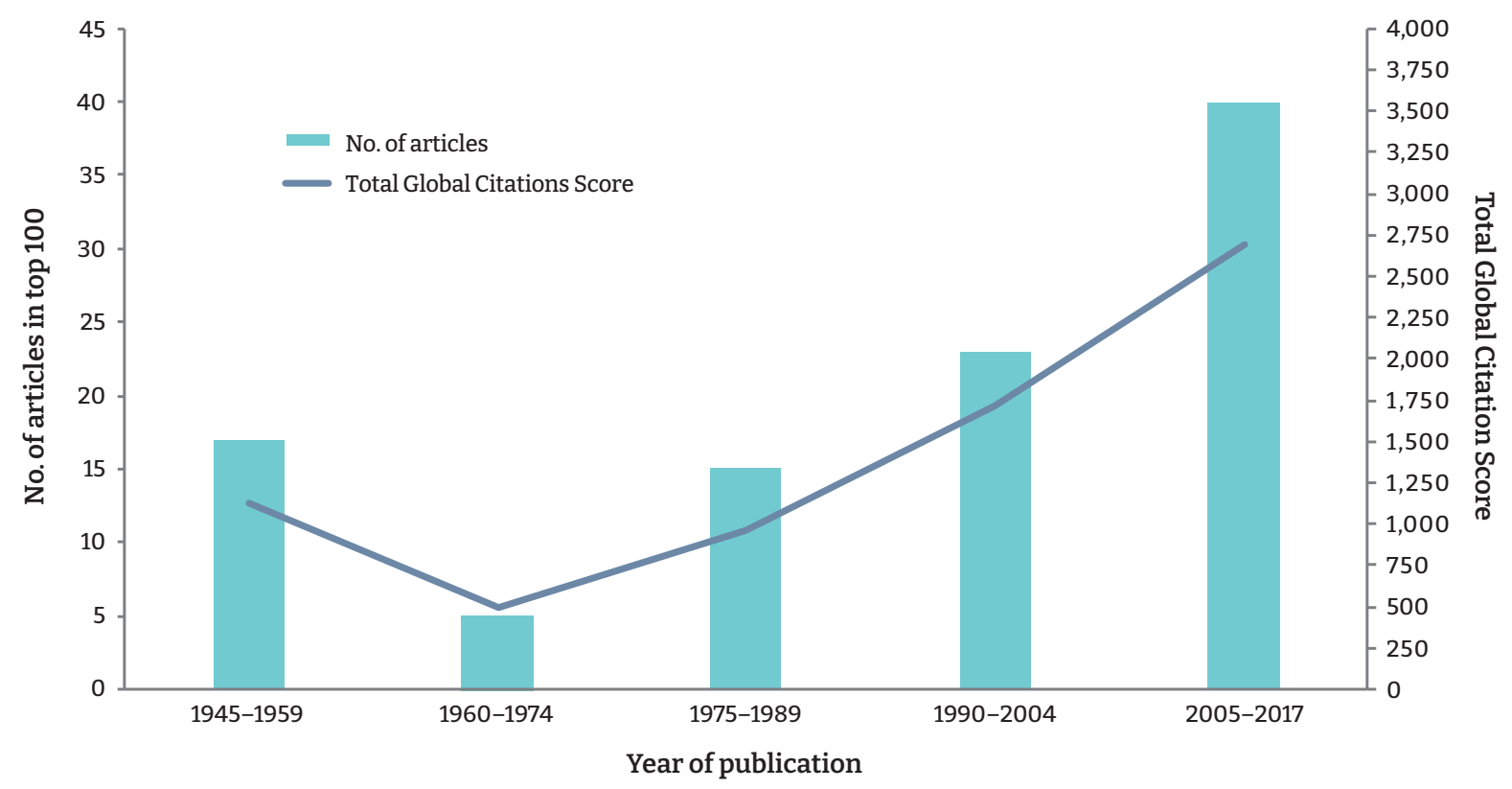

Figure 1. Graph showing the number of scrub typhus articles and Total Global Citations Score by year. 
shown in Table 1. These 14 journals altogether published 65 of the 100 top-cited ST articles. The American Journal of Tropical Medicine and Hygiene, with 14 articles ( $n$ ), a median citations per article (MCA) of 55.0, and an IQR of 39 was the most represented journal, followed by Clinical Infectious Diseases ( $n, 7$; MCA, 68.0; IQR, 77), and Infection and Immunity (n, 7; MCA, 59.0; IQR, 44). Notably, The Lancet, a journal with a JIF of over 60, published only 3 of the 100 top-cited articles on ST.

The earliest top-cited article was published in 1969 in Transactions of the Royal Society of Tropical Medicine and Hygiene, which published another one of the top 100 articles as recently as 2004.

\section{Authorship and Institution Analysis}

We identified 392 authors who contributed to the 100 topcited articles. In terms of authorship, 13 authors contributed to 5 or more of the 100 top-cited articles on ST. The most productive author was Paris (h-index, 10; MCA, 73; IQR, 70), with 4 first-authorships and 10 articles in the top 100 (Table 2). Thirteen institutions contributed 3 or more of the top 100 articles, with Mahidol University in Thailand being the most prolific (h-index, 14; MCA, 60; IQR, 40), followed by the University of Oxford in England (h-index, 10; MCA, 60; IQR, 40) (Table 3).

\section{Country of Origin}

The countries with 2 or more top-cited articles, in terms of both single-country publications (intra-country collaborations), and multi-country publications (inter-country collaborations), are presented in Figure 2. The 100 most-cited ST articles originated from 21 countries, of which 9 countries originated more than 2 articles. The United States (US) was the most productive country with 21 articles, followed by South Korea and Thailand, both with 12 articles.

\section{Keyword Analysis}

The evaluation of keywords in terms of their frequency is shown in Figure 3. The analysis of author's keywords (Figure $3 \mathrm{~A}$ ) revealed the most popular themes in the diverse literature on ST. We found that 'scrub typhus' $(n=14)$, ' $O$. tsutsugamushi' ( $n=10)$, 'Rickettsia' $(n=4)$, 'children' $(n=2)$, and 'clinical manifestations' $(n=2)$ were the most frequent abstract keywords (Figure 3B). Among the top 100 KeyWords Plus results (Figure 3C) were 'doxycycline,' 'diagnosis,' 'polymerase chain reaction,' 'infection,' and 'outbreak,' among others.

\section{Bibliographic Coupling Analysis}

We used VOSviewer software to explore the bibliographic coupling (BC) between the authors, countries, and institutions that contributed to the 100 top-cited articles on ST (Figure 4). For BC analysis of authors, a minimum of 2 instances of authorship was required to meet the search criteria, and 72 authors reached this threshold. The top authors based on the number of links (L) and total link strength (TLS) were Paris (L, 70; TLS, 3,982), followed by Richards (L, 71; TLS, 3,019), Dasch (L, 69; TLS, 1,379), and Shirai (L, 65; TLS, 1,300) (Figure 4A). For the BC analysis between

Table 1. Journals that published at least 2 of the 100 most-cited scrub typhus papers

\begin{tabular}{|c|c|c|c|c|c|c|c|c|}
\hline SCR & $\begin{array}{l}\text { Source journal } \\
\qquad(n=49)\end{array}$ & $\begin{array}{l}\text { Articles } \\
\text { in top } 100\end{array}$ & TGCS (R) & MCA & IQR & 2019 IF & Quartile $^{\text {a) }}$ & Publication $^{\text {b) }}$ \\
\hline $1 \mathrm{st}$ & $\begin{array}{l}\text { American Journal of Tropical Medicine and } \\
\text { Hygiene }\end{array}$ & 14 & $965(1)$ & 55.0 & 39 & 2.126 & Q2 & $1952-2013$ \\
\hline 2nd & Clinical Infectious Diseases & 7 & $627(2)$ & 68.0 & 77 & 8.313 & Q1 & 1997-2009 \\
\hline $3 r d$ & Infection and Immunity & 7 & $461(3)$ & 59.0 & 44 & 2.970 & Q2 & 1967-1982 \\
\hline 4th & Journal of Clinical Microbiology & 6 & $277(7)$ & 42.5 & 14 & 5.897 & Q1 & 1977-2010 \\
\hline 5th & American Journal of Hygiene & 5 & $334(4)$ & 69.0 & 10 & NA & NA & $1945-1952$ \\
\hline 6th & Plos Neglected Tropical Diseases & 5 & $315(5)$ & 63.0 & 11 & 3.885 & Q1 & $2011-2017$ \\
\hline 7th & Emerging Infectious Diseases & 4 & $235(10)$ & 27.5 & 38 & 6.259 & Q1 & 2006-2016 \\
\hline 8th & $\begin{array}{l}\text { Transactions of the Royal Society of Tropical } \\
\text { Medicine and Hygiene }\end{array}$ & 4 & $258(9)$ & 55.5 & 23 & 1.868 & Q3 & 1969-2004 \\
\hline 9th & The Lancet & 3 & $314(6)$ & 84.0 & 114 & 60.390 & Q1 & $1996-2020$ \\
\hline 10th & Antimicrobial Agents and Chemotherapy & 2 & $139(11)$ & 69.5 & 19 & 4.904 & Q1 & 1995-2007 \\
\hline 11th & Current opinion in Infectious Diseases & 2 & $275(8)$ & 137.5 & 179 & 4.577 & Q1 & $2003-2016$ \\
\hline 12th & International Journal of Infectious Diseases & 2 & $112(13)$ & 56.0 & 26 & 3.202 & Q2 & 2013-2014 \\
\hline 13th & Japanese Journal of Infectious Diseases & 2 & $112(13)$ & 56.0 & 8 & 3.202 & Q4 & $2005-2006$ \\
\hline 14th & Tropical Doctor & 2 & $118(12)$ & 59.0 & 38 & 0.523 & Q4 & $2006-2010$ \\
\hline
\end{tabular}

SCR, Standard Competition Ranking; TGCS, Total Global Citation Score; MCA, median citations per article; IQR, interquartile range; IF, impact factor; NA, not available.

a) Journal quartile range. ${ }^{\text {b)}}$ Time span: publication year for reported authors. 
Table 2. Authors who contributed to at least 5 articles of the 100 most-cited scrub typhus papers

\begin{tabular}{|c|c|c|c|c|c|c|c|c|c|}
\hline \multirow{2}{*}{ SCR } & \multirow{2}{*}{$\begin{array}{l}\text { Author } \\
(n=392)\end{array}$} & \multirow{2}{*}{ h-index } & \multicolumn{3}{|c|}{ Authorship position } & \multirow{2}{*}{ TGCS } & \multirow{2}{*}{ MCA } & \multirow{2}{*}{ IQR } & \multirow{2}{*}{ Publication year } \\
\hline & & & First & Second & Third or last & & & & \\
\hline $1 \mathrm{st}$ & Paris DH & 10 & 4 & 1 & 5 & 789 & 73 & 70 & $2007-2017$ \\
\hline $3 r d$ & Smadel JE & 8 & 6 & 2 & 0 & 545 & 67 & 29 & $1948-1952$ \\
\hline 4th & Blacksell SD & 7 & 2 & 1 & 4 & 518 & 57 & 80 & $2006-2016$ \\
\hline 5th & Abraham OC & 6 & 0 & 2 & 4 & 365 & 56 & 37 & 2006-2015 \\
\hline 7th & Shiral A & 6 & 1 & 3 & 2 & 397 & 64 & 43 & $1976-1983$ \\
\hline 8th & Chierakul W & 5 & 1 & 2 & 2 & 259 & 44 & 15 & 2004-2011 \\
\hline 9th & Dasch GA & 5 & 1 & 0 & 4 & 368 & 50 & 9 & 1979-2006 \\
\hline 10th & Day NP & 5 & 0 & 0 & 5 & 348 & 57 & 35 & 2007-2012 \\
\hline 11th & Richards AL & 5 & 1 & 1 & 3 & 434 & 55 & 24 & 1997-2016 \\
\hline
\end{tabular}

SCR, Standard Competition Ranking; h-index, Hirsh-index; TGCS, Total Global Citation Score; MCA, median citations per article; IQR, interquartile range.

a) Time span: publication year for reported authors.

Table 3. Institutions originating 3 or more of the 100 most-cited scrub typhus papers

\begin{tabular}{|c|c|c|c|c|c|c|c|c|}
\hline SCR & Institution, country $(n=129)$ & $\begin{array}{c}\text { Article } \\
\text { in top } 100^{\text {a) }}\end{array}$ & TLSC & TGCS & NA & MCA & IQR & Publication $^{\text {b) }}$ \\
\hline $1 \mathrm{st}$ & Mahidol University, Thailand & 14 & 35 & 1,004 & 100 & 60 & 40 & 2004-2017 \\
\hline 2nd & University of Oxford, England & 10 & 31 & 715 & 72 & 60 & 40 & 2004-2017 \\
\hline $3 r d$ & University School of Nashville, USA & 8 & 52 & 627 & 72 & 58 & 42 & 1973-2011 \\
\hline 4th & Walter Reed Army Institute of Research, USA & 8 & 39 & 550 & 69 & 68 & 38 & $1976-2000$ \\
\hline 5 th & Christian Medical College \& Hospital, India & 6 & 17 & 365 & 46 & 58 & 37 & 2006-2015 \\
\hline 6th & Chosun University, South Korea & 4 & 13 & 252 & 32 & 57 & 31 & $2006-2010$ \\
\hline 7th & Seoul National University, South Korea & 4 & 15 & 319 & 20 & 59 & 78 & 1993-2001 \\
\hline 8th & Armed Forces Research Institute of Medical Sciences, USA & 3 & 16 & 332 & 17 & 58 & 180 & $2000-2003$ \\
\hline 9th & Haenam General Hospital, South Korea & 3 & 13 & 161 & 28 & 48 & 19 & 2006-2007 \\
\hline 10th & Mahosot Hospital, Laos & 3 & 7 & 270 & 13 & 83 & 61 & 2010-2017 \\
\hline 11 th & Seonam University, South Korea & 3 & 13 & 161 & 28 & 48 & 19 & 2006-2007 \\
\hline 12th & University of Texas Medical Branch, USA & 3 & 6 & 230 & 33 & 68 & 74 & 2010-2017 \\
\hline 13th & Walter Reed National Military Medical Center, USA & 3 & 20 & 194 & 32 & 60 & 34 & $1995-2000$ \\
\hline
\end{tabular}

SCR, Standard Competition Ranking; TLSC, Total Local Citation Score; TGCS, Total Global Citation Score; NA, number of authors; MCA, median citations per article; IQR, interquartile range.

a)Total number of citations. ${ }^{\text {b) }}$ Time span: publication year for reported authors.

countries, at least 2 must have originated in a country for it to be included in the analysis. Thirteen countries met this threshold and were visualized as 3 clusters. The country with the highest TLS was Thailand (L, 75; TLS, 3,631), followed by the US (L, 2,328; TLS, 3,468), England (L, 865; TLS, 2,667), South Korea (L, 793; TLS, 1,239), India (L, 601; TLS, 1,188), and Laos (L, 411; TLS, 1,137) (Figure 4B). For the BC analysis between institutions, at least 2 articles were required to have originated from an institution to be included, which resulted in 30 institutions visualized as 4 clusters. Mahidol University had the highest number of collaborations (L, 29; TLS, 2,783), followed by Naval Medical Research Center (L,
29; TLS, 999), and Christian Medical College \& Hospital (L, 29; TLS, 810) (Figure 4C).

\section{Funding Agencies}

As shown in Figure 1, the annual number of articles on ST increased gradually, from just 23 in the 14-year range of 1990 to 2004, to 40 in the 12 -year range of 2005 to 2017. This phenomenon may be the result of increased financial support from various funding sources such as Wellcome Trust, Global Emerging Infections Surveillance and Response System, and the United States Army Medical Research and Materiel Command and Naval Medical 


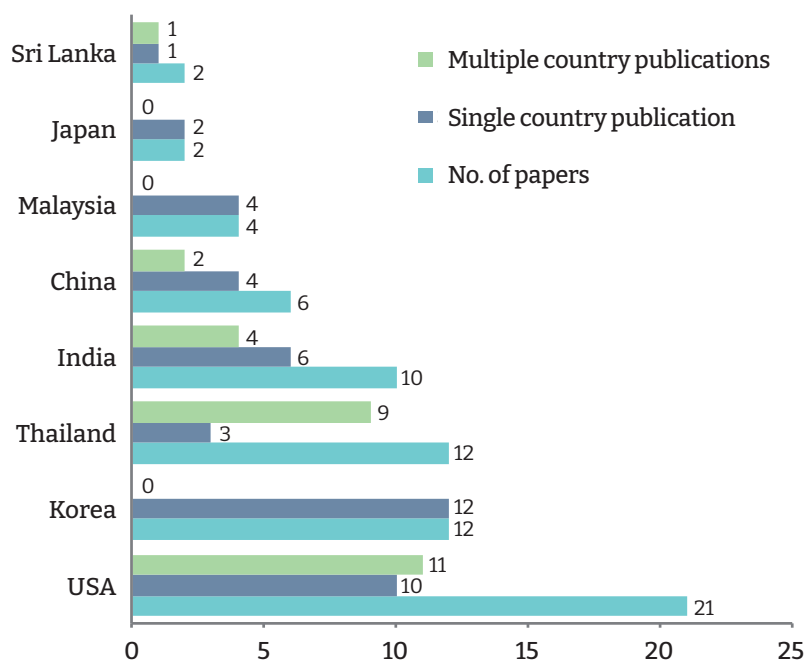

Figure 2. Most productive and influential countries on scrub typhus research with 2 or more articles, including both single-country publications (intra-country collaboration) and multiple-country publications (intercountry collaboration).

Research Center (Table S3). These, as well as other funding institutions, supported a total of 20 of the 100 top-cited ST articles.

\section{Factors Influencing Citations Scores}

The factors that determined the number of citations of the top-cited articles on ST are presented in Table 4. We explored possible correlations between the number of citations and study variables such as number of authors, number of institutions, years since publication, JIF, and number of countries. We observed a strong positive association between the citation score and number of years since publication $(r=0.869, p<0.001)$, number of authors and number of citations $(\mathrm{r}=0.668, p<0.001)$, number of institutions and citation score $(r=0.692, p<0.001)$, as well as number of countries and number of citations $(r=0.963$, $p<0.001)$. However, there was not a significant correlation between the JIF and citations $(r=0.158, p=0.277)$.

\section{Discussion}

The study provided insights into the trends of the most influential ST publications over the past $100-120$ years using comprehensive bibliometric tools [10,17,18]. All 100 top-cited ST publications were written in English, and the majority were from US institutions, which produced more articles than those of any other country [18]. This may be because the US has a higher gross national product than other countries, which enables US researchers to be the more productive in ST research. Of the 100 most-cited articles, a total of 21 were from the US, followed by 12 articles from South Korea and 12 articles from Thailand [22].

A total of 392 authors from 21 countries contributed to the 100 top-cited articles with a citation range of 39 to 227 (MCA, 56; IQR, 35.5). The top 3 most-productive authors in this study were Paris, Osterman, and Smadel, who collectively contributed 27 articles as the first, second, or third or last author, and accrued a total of 1,913 citations. Overall, 13 key authors contributed to $82 \%$ of the top 100 articles in the ST field and were referred to in 5,996 citations during our study period. This number of citations, however, is less than is reported in some other medical fields such as islet transplantation, where the citation count ranged from 146 to 2,988 [23]. The difference in citation count could be a result of the number of researchers contributing to specific medical fields [24], or possibly due to there being less research interest in the field of ST compared to other diseases [11].

In addition, open access status may be another important factor in attracting citations, since open access articles are freely accessed and tend to be more cited than self-archived or closed access articles [24,25].

The most-cited and influential paper on ST was a review that discussed several key aspects of ST and tropical rickettsioses, published in 2003 in the Journal of Current Opinion in Infectious Diseases. The article indicated that modern developments in molecular taxonomic methods have led to a reclassification of rickettsial disease, and it estimated that 1 billion people are at risk, with 1 million cases occurring annually. Additionally, the review noted that the lack of professional or non-specialist physicians and disease misdiagnoses raise more questions than answers, implying the need for much more research on ST. As a result, this article became the most influential within the field, with 227 citations [1].

The second most-cited article received 216 citations and focused on the geographic distribution of phenotypic and genotypic variants of $O$. tsutsugamushi, published in Clinical Infectious Diseases as a review article. Our study indicates that a minority of articles on ST are responsible for the majority of citations, which is a similar result to other published bibliometric studies [18].

Other top-cited articles discussed key aspects and discoveries in ST research such as antibiotic use in the treatment of ST, including, for example, articles on chloromycetin [26], doxycycline compared to azithromycin [27], and chloramphenicol and tetracycline to treat patients with ST infection [28].

As shown in this study, there was a significant correlation between the number of citations and the number of years 

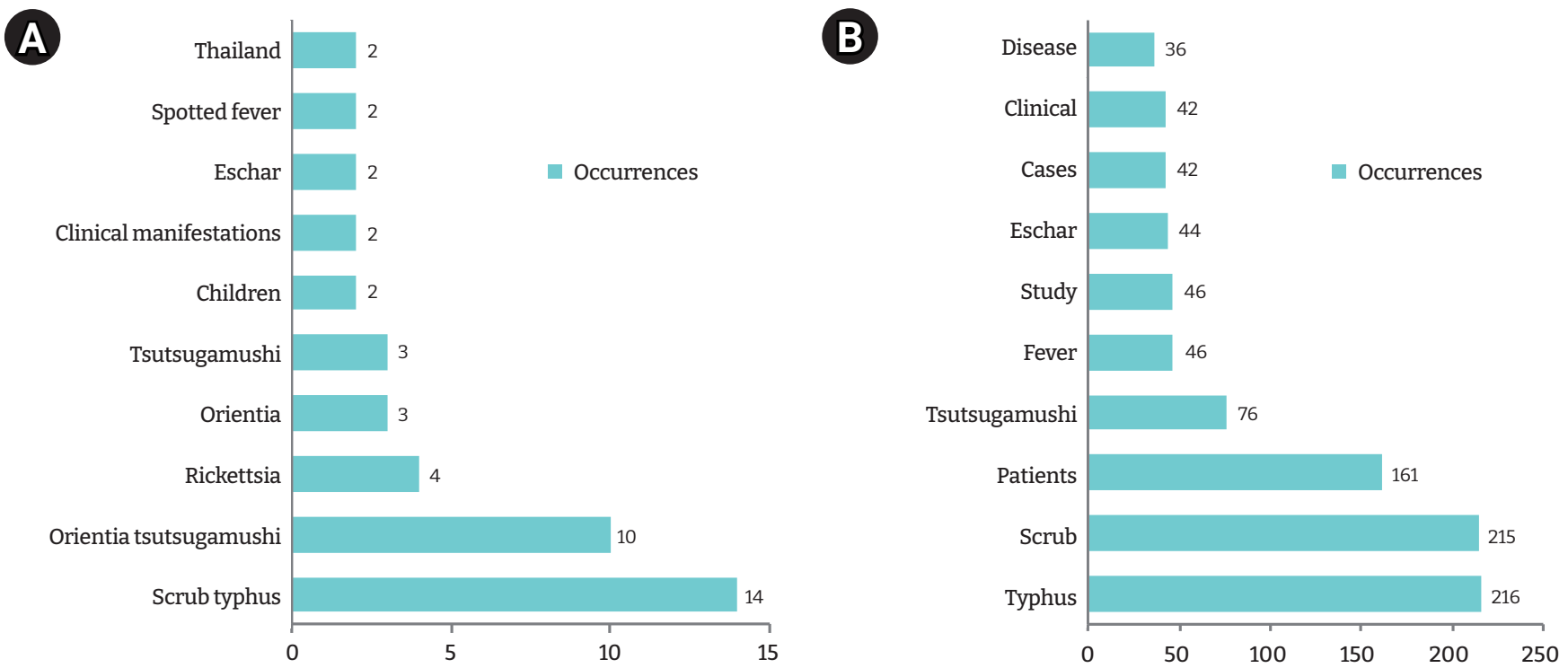

C

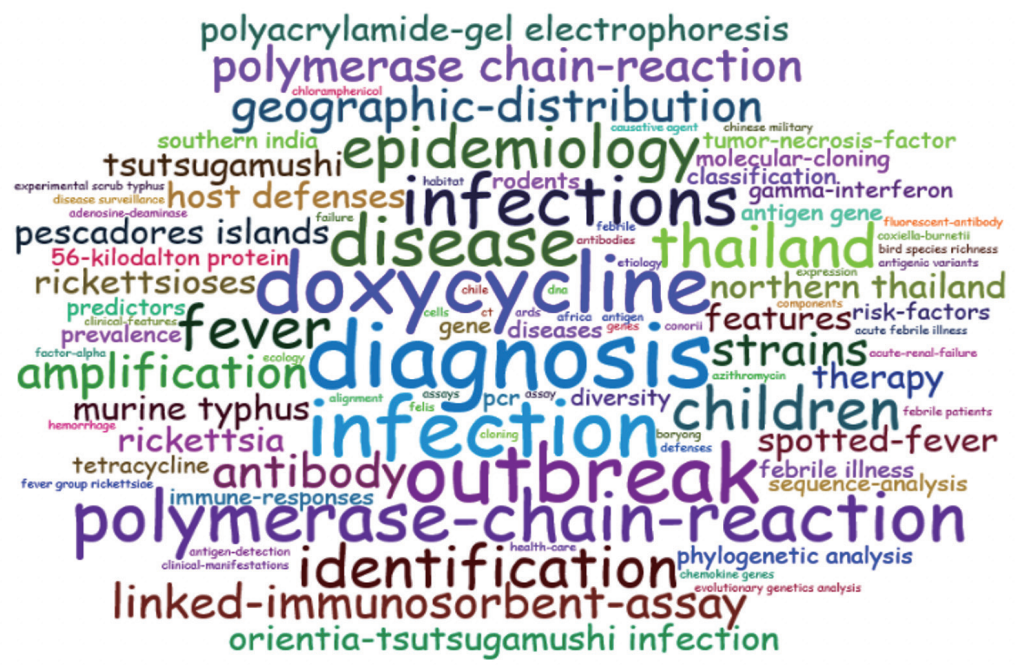

Figure 3. Keyword occurences of (A) author's keywords and (B) abstract keywords. (C) Word cloud visualization of KeyWords Plus.

since publication. This finding differs from bibliometric findings from other fields such as gastroenterology and hepatology, for which no significant correlations were found between the number of citations and the number of years since publication [17]. In addition, significant correlations were found between the number of institutions involved, the number of countries, and the number of citations. Similar associations were previously described by Azer and Azer [17] in 2016.

Across the 49 different journals that published ST research, the top-cited articles were published not only in journals with a high impact factor, but also in lower-impact journals [29]. Previous bibliometric studies have shown that the impact factor of particular journals is considered the best indicator used for journal citations. Our study also demonstrated that the JIF does not necessarily influence the citation performance of articles, and it is possible that highly cited articles can be published in journals with a low JIF. Examples of such articles are included in our references [28,30-34]. This indicates that most researchers focused on other factors aside from JIF when publishing ST research.

Regarding journal productivity, the American Journal of Tropical Medicine and Hygiene published the most papers related to ST. This can be seen as a pioneering journal that gave ST research particular attention. Furthermore, most of these journals belonged to the first and second quartiles, 

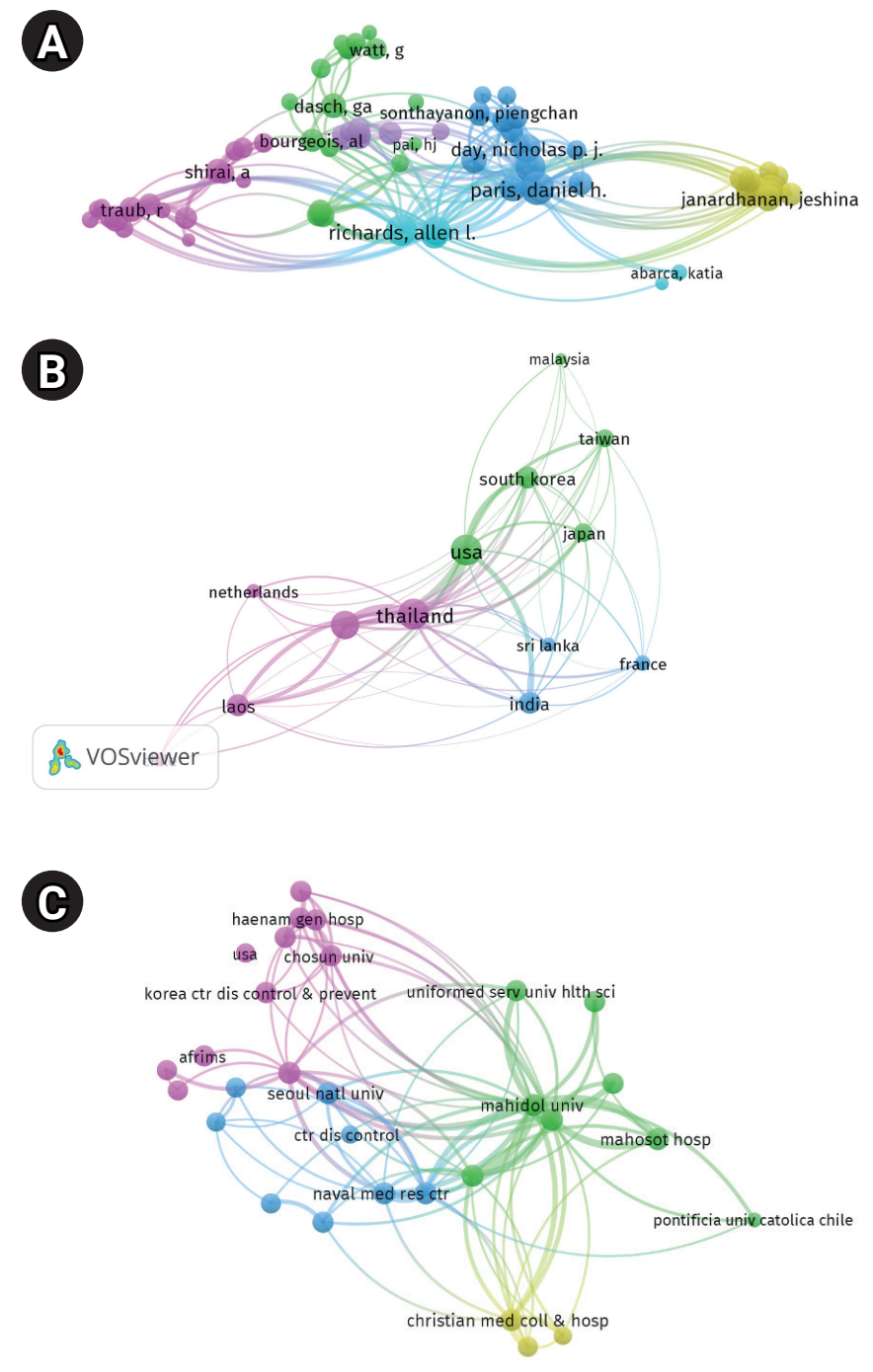

Figure 4. Bibliographic coupling of authors $(A)$, countries (B), and organizations (C) that contributed to the 100 most-cited articles in scrub typhus research.

which confirms the quality of the ST research being published by these journals.

Our study shows that only $20 \%$ of the top-cited articles on ST reported funding. As previously reported, funding is a potential mechanism to encourage and increase productivity within a given field. Thus, to enable researchers to carry out more ST research in key fields such as the development of vaccines for ST prevention and control, increased funding is crucial [18].

The analysis of keywords indicated that the top-cited articles on ST covered various key aspects of ST including diagnosis, diseases symptoms, infection, its effect on children, clinical treatments, and advance diagnostic methods such as the use of polymerase chain reaction. These keywords
Table 4. Associations between citations and study variables

\begin{tabular}{lccr}
\hline \multicolumn{1}{c}{ Characteristic } & Research & Spearman $\mathbf{r}$ & $\boldsymbol{p}$ \\
\hline No. of authors & 392 & 0.668 & $<0.001$ \\
No. of institutions & 129 & 0.692 & $<0.001$ \\
No. of years since publication & 41 & 0.869 & $<0.001$ \\
Journal impact factor (2019) & 49 & 0.158 & 0.277 \\
No. of countries & 21 & 0.963 & $<0.001$ \\
\hline
\end{tabular}

highlighted future trends and popular topics in ST research.

The institutions which produced the most research were primarily located in Asian countries such as Thailand, India, and South Korea, among others. This reflects the burden of the disease, as ST is endemic in the Asia-Pacific region and therefore influences research efforts in these countries. However, contributions from other regions and countries such as the US show the worldwide scope of ST research.

In terms of link strength, the 100 most-cited studies were produced through collaboration among different authors, countries, and institutions. From one perspective, this could imply the importance of collaborative efforts and partnerships in achieving research advances in the field and making them available in well-known databases. The results of the co-authorship network show the current state of collaboration and the most influential authors who produced the top-cited studies on ST. Evidence from the network analysis reveals a significant level of collaboration among authors in the field, visualized in 3 broad clusters. Collaboration is vital, as it enables the sharing of knowledge and scientific findings such as genome sequences and possible development of an ST vaccine.

\section{Strengths and Limitations}

By using bibliometric and visualization analyses, the present study furnishes deep insights into the 100 most-cited articles on ST. However, some limitations must be addressed. First, we analyzed results from a single database (WoS). Thus, there is a possibility of having missed key top-cited articles that are only available in other databases such as Scopus, PubMed, and Google Scholar. Therefore, the results of our assessment might not be fully comprehensive. Second, we only included studies published in the English language and overlooked studies published in other languages, which may themselves be highly cited. Third, it was not possible to search by category or to search for the top-cited articles in relevant fields, either manually or from the WoS website.

\section{Conclusion}

This study is the first bibliometric analysis of the 100 most- 
cited articles on ST indexed in WoS. We found that the trend and growth of most-cited articles on ST had a significant increase. The majority of articles originated in the US and various Asian countries, which showed a significant contribution to new research in the field, with strong research collaboration across authors, countries, and organizations. The American Journal of Tropical Medicine and Hygiene was the most prolific publisher of ST articles in terms of journals. Mahidol University in Thailand led the list of the most prolific institutions producing ST research. An article's citation count was significantly correlated with the number of authors, institution, years since publication, and countries involved, but not with JIF. In addition, only $20 \%$ of articles received funding, so adequate funding and institutional capacity-building are required to increase the impact of future developments in ST research. These findings may provide new insights for researchers and health policy-makers, thereby enabling a better understanding of the ST disease globally.

\section{Supplementary Material}

Table S1. Characteristics of metadata; Table S2. The 100 mostcited articles on scrub typhus; Table S3. The funding agencies that contributed to the 100 most-cited scrub typhus articles. Supplementary data is available at https://doi.org/10.24171/ j.phrp.2021.12.2.10.

\section{Notes}

\section{Ethics Approval}

Not applicable.

\section{Conflicts of Interest}

The authors have no conflicts of interest to declare.

\section{Funding}

The study was fully funded by a Jiangsu Provincial Social Science Foundation Grant (13TYA001).

\section{Availability of Data}

All the data supporting the results of this study are included in the article and the supplementary file. The raw data on ST can also be directly obtained from the Web of Science Core Collection (https:// webofknowledge.com/) using the appropriate search query.

\section{Additional Contributions}

The authors are grateful to the Southeast University electronic library for providing facilities and library resources for accessing the routine surveillance data used in this study.

\section{References}

1. Watt G, Parola P. Scrub typhus and tropical rickettsioses. Curr Opin Infect Dis 2003;16:429-36.

2. Beran GW, Steele JH, Benenson AS, et al. Handbook of zoonoses, section a: bacterial, rickettsial, chlamydial and mycotic. 2nd ed. Boca Raton (FL): CRC Press; 1994.

3. Kelly DJ, Fuerst PA, Ching WM, et al. Scrub typhus: the geographic distribution of phenotypic and genotypic variants of Orientia tsutsugamushi. Clin Infect Dis 2009;48 Suppl 3:S203-30.

4. Li Z, Xin H, Sun J, et al. Epidemiologic changes of scrub typhus in China, 1952-2016. Emerg Infect Dis 2020;26:1091-101.

5. Rosenberg R. Drug-resistant scrub typhus: paradigm and paradox. Parasitol Today 1997;13:131-2.

6. Mokta J, Ranjan A, Mokta K. Early clinical suspicion and early use of doxycycline reduces scrub typhus associated complications. J Assoc Physicians India 2019;67:26-7.

7. Hengbin G, Min C, Kaihua T, et al. The foci of scrub typhus and strategies of prevention in the Spring in Pingtan Island, Fujian Province. Ann N Y Acad Sci 2006;1078:188-96.

8. van Nunen K, Li J, Reniers G, et al. Bibliometric analysis of safety culture research. Saf Sci 2018;108:248-58.

9. Wang B, Pan SY, Ke RY, et al. An overview of climate change vulnerability: a bibliometric analysis based on Web of Science database. Nat Hazards 2014;74:1649-66.

10. Ramos MB, Teixeira MJ, Preul MC, et al. A bibliometric study of the most cited reports in central nervous system arteriovenous malformations. World Neurosurg 2019;129:261-8.

11. Feasey N, Wansbrough-Jones M, Mabey DC, et al. Neglected tropical diseases. Br Med Bull 2010;93:179-200.

12. Rights FL, Smadel JE. Studies on scrub typhus; tsutsugamushi disease; heterogenicity of strains of R. tsutsugamushi as demonstrated by cross-vaccination studies. J Exp Med 1948;87:339-51.

13. Premaratna R, Blanton LS, Samaraweera DN, et al. Genotypic characterization of Orientia tsutsugamushi from patients in two geographical locations in Sri Lanka. BMC Infect Dis 2017;17:67.

14. Shuaib W, Khan MS, Shahid $\mathrm{H}$, et al. Bibliometric analysis of the top 100 cited cardiovascular articles. Am J Cardiol 2015;115:972-81.

15. Malhotra K, Saeed O, Goyal N, et al. Top-100 highest-cited original articles in ischemic stroke: a bibliometric analysis. World Neurosurg 2018;111:e649-60.

16. Harzing AW, Alakangas S. Google Scholar, Scopus and the Web of Science: a longitudinal and cross-disciplinary comparison. Scientometrics 2016;106:787-804.

17. Azer SA, Azer S. Bibliometric analysis of the top-cited gastroenterology and hepatology articles. BMJ Open 2016;6:e009889.

18. Paniagua Cruz A, Zhu KY, Ellimoottil C, et al. Characterizing the benign prostatic hyperplasia literature: a bibliometric analysis. Urology 2020;136:202-11.

19. Ellegaard O, Wallin JA. The bibliometric analysis of scholarly production: how great is the impact? Scientometrics 2015;105:1809-31.

20. Aria M, Cuccurullo C. bibliometrix: an R-tool for comprehensive science mapping analysis. J Informetr 2017;11:959-75.

21. van Eck NJ, Waltman L. Software survey: VOSviewer, a computer program for bibliometric mapping. Scientometrics 2010;84:523-38. 
22. Mainwaring A, Bullock N, Ellul T, et al. The top 100 most cited manuscripts in bladder cancer: a bibliometric analysis (review article). Int J Surg 2020;75:130-8.

23. Pu QH, Lyu QJ, Liu H, et al. Bibliometric analysis of the top-cited articles on islet transplantation. Medicine (Baltimore) 2017;96:e8247.

24. Eysenbach G. Citation advantage of open access articles. PLoS Biol 2006;: :e157

25. Antelman K. Do open-access articles have a greater research impact? Coll Res Libr 2004;5:372-82.

26. Smadel JE, Woodward TE. Chloromycetin in the treatment of scrub typhus. Med Bull US Army Eur 1948;5:32.

27. Phimda K, Hoontrakul S, Suttinont C, et al. Doxycycline versus azithromycin for treatment of leptospirosis and scrub typhus. Antimicrob Agents Chemother 2007;51:3259-63.

28. Sheehy TW, Hazlett D, Turk RE. Scrub typhus: a comparison of chloramphenicol and tetracycline in its treatment. Arch Intern Med 1973;132:77-80
29. Chrispal A, Boorugu H, Gopinath KG, et al. Scrub typhus: an unrecognized threat in South India-clinical profile and predictors of mortality. Trop Doct 2010;40:129-33.

30. Smadel JE, Ley HL Jr, Diercks FH, et al. Immunity in scrub typhus: resistance to induced reinfection. AMA Arch Pathol 1950;50:847-61.

31. Chattopadhyay S, Richards AL. Scrub typhus vaccines: past history and recent developments. Hum Vaccin 2007;3:73-80.

32. Bozeman FM, Elisberg BL. Serological diagnosis of scrub typhus by indirect immunofluorescence. Proc Soc Exp Biol Med 1963;112:56873.

33. Jackson EB, Smadel JE. Immunization against scrub typhus: II. preparation of lyophilized living vaccine. Am J Hyg 1951;53:326-31.

34. Settle EB, Pinkerton H, Corbett AJ. A pathologic study of tsutsugamushi disease (scrub typhus) with notes on clinicopathologic correlation. J Lab Clin Med 1945;30:639-61. 